\title{
Review Article \\ Unraveling Plant Responses to Bacterial Pathogens through Proteomics
}

\author{
Tamara Zimaro, ${ }^{1}$ Natalia Gottig, ${ }^{1}$ Betiana S. Garavaglia, ${ }^{1}$ \\ Chris Gehring, ${ }^{2}$ and Jorgelina Ottado ${ }^{1}$
${ }^{1}$ Molecular Biology Division, Instituto de Biología Molecular y Celular de Rosario, Consejo Nacional de Investigaciones Científicas y Técnicas, Facultad de Ciencias Bioquímicas y Farmacéuticas, Universidad Nacional de Rosario, Suipacha 531, Rosario S2002LRK, Argentina
${ }^{2}$ Division of Chemistry, Life Science and Engineering, King Abdullah University of Science and Technology, Thuwal 23955-6900, Saudi Arabia

Correspondence should be addressed to Jorgelina Ottado, ottado@ibr.gov.ar

Received 31 May 2011; Revised 24 August 2011; Accepted 2 September 2011

Academic Editor: Francesca Cutruzzolà

Copyright (C) 2011 Tamara Zimaro et al. This is an open access article distributed under the Creative Commons Attribution License, which permits unrestricted use, distribution, and reproduction in any medium, provided the original work is properly cited.

\begin{abstract}
Plant pathogenic bacteria cause diseases in important crops and seriously and negatively impact agricultural production. Therefore, an understanding of the mechanisms by which plants resist bacterial infection at the stage of the basal immune response or mount a successful specific R-dependent defense response is crucial since a better understanding of the biochemical and cellular mechanisms underlying these interactions will enable molecular and transgenic approaches to crops with increased biotic resistance. In recent years, proteomics has been used to gain in-depth understanding of many aspects of the host defense against pathogens and has allowed monitoring differences in abundance of proteins as well as posttranscriptional and posttranslational processes, protein activation/inactivation, and turnover. Proteomics also offers a window to study protein trafficking and routes of communication between organelles. Here, we summarize and discuss current progress in proteomics of the basal and specific host defense responses elicited by bacterial pathogens.
\end{abstract}

\section{Introduction}

Plants are constantly exposed to microbes and counteract them by activating an innate immune response, including mechanisms of basal and race-specific resistance [1]. A contemporary view of plant immunity proposes a model where the first line of active plant defense involves plant pathogen recognition through specific receptors, the pattern recognition receptors (PRRs) that in turn recognize pathogenassociated molecular patterns (PAMPs). This response mechanism is termed PAMP-triggered immunity (PTI). Classical examples of bacterial PAMPs are structural molecules such as bacterial flagellins, peptidoglycans, and lipopolysaccharides [2-5]. Another example of a PAMP is the harpin protein HrpZ of the bacterial pathogen Pseudomonas syringae [6]. PTI involves signaling through the second messenger cGMP $[7,8]$, mitogen-activated protein kinases (MAPKs), $\mathrm{Ca}^{2+}$ and $\mathrm{H}^{+}$influx, early accumulation of reactive oxygen species (ROS), cell wall thickening leading to papillae formation, and altered expression of pathogenesis-related (PR) genes [1,9]. Successful pathogens are able to overcome PTI by means of secreted effectors that suppress PTI responses, resulting in effector-triggered susceptibility (ETS). Such effectors are injected by pathogenic bacteria into the host cytoplasm through their type three protein secretion system (TTSS). Effectors promote pathogenicity, and the TTSS is essential for the development of disease symptoms and bacterial multiplication. Because of their collective action, effectors are hypothesized to alter plant homeostasis in susceptible hosts and thereby sustain pathogen growth [10]. Such effector responses are caused, for example, by $P$. syringae effectors AvrPto, AvrRpt2, and AvrRpm1 that inhibit defense responses elicited by PAMP recognition [11, 12]. Bacterial effector proteins such as members of the Xanthomonas 
AvrBs3 effector family have also been implicated in activating plant transcription $[13,14]$. The effector proteins AvrB, AvrRpt2, AvrPphB, HopPtoK, and AvrPphE have been shown to modulate host signaling via salicylic acid (SA), jasmonic acid (JA), and ethylene, all of which have a role in plant defense [15]. In response to this threat, plants have evolved $\mathrm{R}$ proteins which typically are intracellular receptor proteins of the NB-LRR type that recognize effectors in the cytoplasm thereby activating the so-called effector-triggered immunity (ETI). Typically, the ability to trigger ETI is pathogen strain or race-specific and is associated with programmed cell death, a response which is referred to as the hypersensitive response (HR), and systemic acquired resistance (SAR) in the host $[1,9]$. Some of the best characterized $\mathrm{R}$ proteins are RPS2, RPM1, and RPS5. These Arabidopsis R proteins confer resistance to $P$. syringae carrying the bacterial effectors AvrRpt2, AvrRpm1/AvrB, and AvrPphB, respectively, [16]. In a recent report, different experimental data are integrated and novel interactions in plant immunity responses are proposed [17]. Regarding the distinction of PAMPs and effectors, one aspect of the current model considers that while PAMPs are widely conserved, effectors are specific to single or only a few related species [9]. However, several effector proteins are also widespread and may be considered as PAMPs [17]. Another issue of the contemporary immunity model is that whereas PAMPs are considered essential for microbial fitness and survival, effectors are considered to specifically contribute to virulence. However, several PAMPs also have a role in pathogen virulence; examples include the bacterial flagellum [2] and the HrpZ harpin that play a role in virulence by affecting host membrane integrity [18]. In agreement with the current plant immunity model, PAMP receptors and resistance proteins (PRRs) are evolutionarily ancient surface receptors conserved between species, and most $\mathrm{R}$ proteins typically are intracellular receptors that are evolutionarily relatively young with novel members continuously being discovered [9]. Nevertheless, some of the characterized $\mathrm{R}$ proteins display the typical properties of PRRs and some of them may be relatively recent acquisitions $[19,20]$. Another consideration is that not all effectors are recognized inside host cells and not all extracellular pathogen receptors recognize PAMPs through direct physical interactions [21]. Lastly, as mentioned above, the current model of plant immunity defines that responses of ETI occur more quickly, are more prolonged, and are stronger than those of PTI, suggesting that PTI is a weak variant of ETI [1]. Typically, ETI is associated with an HR and SAR, while PTI is not. However, some PAMPs such as the HrpZ harpin can induce HR in plants [18], demonstrating that the induction of HR can occur in other reponses different from ETI. Additionally, it has been observed that PAMPs also trigger SAR in plants [22]. On the other hand, atypical examples of weak ETI responses were observed with some effector proteins [23]. Taking into account all these considerations, it was proposed that the distinction between PAMPs and effectors, between PAMP receptors and resistance proteins, and therefore also between PTI and ETI cannot be uphold strictly [17]. Instead, it was hypothesized that there is a continuum between PTI and ETI and argued that plant resistance is determined by immune receptors that recognize appropriate ligands to activate defense, the amplitude of which is likely determined by the level required for effective immunity [17].

Worldwide, the diseases caused by pathogens in important crops greatly and negatively impact agricultural production and the environment. Consequently, an understanding of the mechanisms by which plants resist bacterial infection or mount a successful defense response is vital since a better and more detailed understanding of the molecular mechanism of these interactions will eventually enable transgenic approaches that will contribute to minimizing the effects of many of the most devastating diseases. To gain an indepth understanding of plant defense systems, there is a need to identify the diverse and complex signaling cascades and the multiple and interacting biochemical pathways activated by the pathogen. A number of studies addressing this complexity utilizing DNA microarrays or DNA chips have already been carried out with view to investigate the global changes in the transcriptome caused by pathogens [24-26]. However, transcriptional changes are only a part of the response since they do not inform on posttranscriptional and posttranslational processes, protein activation, and turnover. This vital information can come from proteomics that allows monitoring differences in abundance of proteins present at the time of sampling and offers a window to study protein trafficking and routes of communication between organelles. Thus, complementary approaches such as proteome-based expression profiling are needed to obtain a more complete picture of the regulatory elements in plant-pathogen interactions.

Here, we shall concentrate on some of the recent progress made in the use of proteomics to enhance the understanding of basal and specific host defense responses elicited by bacterial pathogens, since a wide range of more general aspects of proteome changes during plant-microbial interactions have been detailed elsewhere [27-29].

The typical experimental design (Figure 1) for evaluating the actual proteome of plants undergoing different types of interactions with pathogenic bacteria consists of a number of sequential steps. Plant material used in proteomics studies includes leaves, roots, and stems as well as calli and cell suspensions. Plant tissue is inoculated with pathogenic bacteria or with bacterial components that constitute PAMPs or elicitors. While bacterial PAMPs trigger a defense response in the plant (PTI), effectors cause disease in susceptible plants (ETS) or trigger ETI mediated by R genes. Different sampling times after-inoculation may be evaluated. Protein samples with appropriate biological replicates obtained are separated in two-dimensional gel electrophoresis (2-DE), generally followed by protein staining. The most frequently used protein stains are Coomassie Brilliant Blue, silver nitrate, Sypro dyes (Orange, Red, Tangerine, and Ruby), Deep Purple, ProQ Emerald (for glycoproteins), ProQ Diamond (for phosphoproteins), CyDyes/DIGE (cyanine dyes/difference in-gel electrophoresis), and immunological detection. Less common methods include negative $\mathrm{Zn}$-stain and radioactive isotopes. For the choice of detection method, important aspects should be taken into account such as 


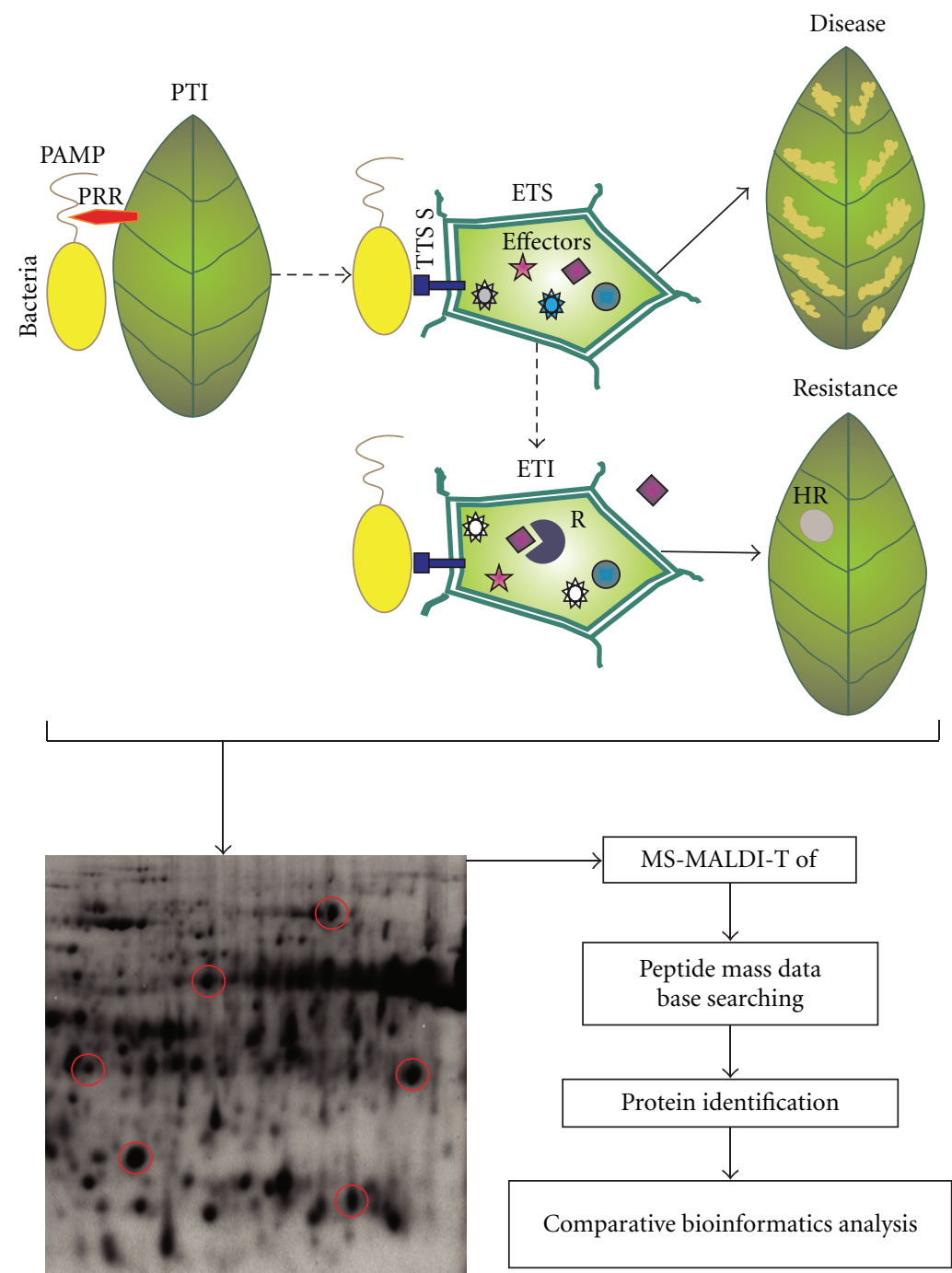

FIgURE 1: When the plant encounters a microorganism, the recognition of bacterial molecular patterns (PAMPs) by plant membrane receptors (PRRs) triggers the PAMP-triggered immunity (PTI). Pathogenic bacteria overcome this defense response by injecting the type three secretion system (TTSS) proteins called effectors that promote pathogenicity. This process is called effector-triggered susceptibility (ETS) and leads to plant disease. Some plants respond to these effectors through the development of R proteins that recognize effectors and activate the so-called effector-triggered immunity (ETI). This is a response associated with a specific programmed cell death called hypersensitive response (HR) that limits pathogen growth and culminates into plant resistance. Protein samples are extracted from tissues of different plant cultivars inoculated with different bacterial strains or bacterial components that trigger the above-mentioned responses. Spots showing differential abundance compared to control treatment (circled in red in the gel picture) are then subjected to subsequent proteomic analysis to resolve response-specific plant protein signatures.

sensitivity (detection limit), dynamic range, linearity, reproducibility, protein-to-protein variability, and compatibility with identification methods [30,31]. Protein spots selected from these gels have to be picked, trypsin digested, and subjected to ionization by electrospray ionization (ESI) or matrix-assisted laser desorption/ionization (MALDI), both coupled to mass spectrometric (MS) analyses. Subsequently, the peptide masses are used to query peptide mass databases in order to identify the proteins. Moreover, multiple fluorescent protein labels used in different ingel electrophoresis (DIGE) offer the possibility to compare the protein composition and abundance of two different samples within a single gel $[32,33]$. Proteins in two samples are labeled in vitro through cysteine or lysine residues using two different fluorescent cyanine dyes differing in their excitation and emission wavelengths, but with an identical relative mass. Labeled samples are then mixed and subjected to 2-DE on the same gel. Differences in the two samples are detected after consecutive excitation with both wavelengths, overlay, and normalization of the images [34]. Even though 2-DE techniques allow high protein separation capacity and the possibility of making large-scale proteinprofiling experiments, issues concerning reproducibility are still a drawback in these procedures. It has been reported 
that proteins with extreme physicochemical properties (size, isoelectric point, and transmembrane domains) and those of low abundance are underrepresented by this method [35]. In order to overcome such difficulties, new techniques based on liquid chromatography (LC) separation of proteins or peptides were developed. These techniques consist of twodimensional liquid-phase chromatography that is based on a high-performance chromatofocusing in the first dimension followed by high-resolution reversed-phase chromatography in the second [36] and one-dimensional 1-DE-nanoscale capillary LC-MS/MS, namely, GeLC-MS/MS, that combines a size-based protein separation with an in-gel digestion of the resulting fractions. Between the LC-based strategies, there is a particular methodology, Multidimensional Protein Identification Technology (MudPIT), that allows the detection of a much larger number of proteins compared to gel-based methods [37].

\section{Proteomics of PAMP-Triggered Immunity}

Plants recognize PAMPs molecules on the surface of the pathogens by pattern recognition receptor proteins that are able to transduce signals that give rise to the innate immune response. A key goal of plant-pathogen interaction research is to decipher how the coordinated changes that cause PTI occur. Proteomics studies have been performed in plants during PAMP-induced responses and they have proven valuable in unraveling the mechanisms that mediate the early events leading to the basal immune response. Two of the most studied PAMPs are the gram-negative bacterial lipopolysaccharide (LPS) and flagellin and its elicitor-active epitope, flg22.

Exhaustive proteomics analyses of Nicotiana tabacum cells elicited with LPS extracted from Burkholderia cepacia were done to identify cellular targets of the signal components induced by this molecule towards understanding the process of LPS perception and subsequent signal transduction $[38,39]$. B. cepacia is not a tobacco pathogen, but the bacteria have a protective effect against subsequent inoculations with the pathogenic fungus Phytophthora nicotianae [40] and thus are considered a good candidate for studying elicitation of the immune response and in particular the proteins involved in LPS-induced signaling. Results obtained with cell suspension cultures were similar to those achieved with leaf discs and intact young leaves and hence provide an experimentally simpler and more reproducible system to do proteomics studies [40]. Qualitative and quantitative changes in phosphoproteins were analyzed using two-dimensional electrophoresis in combination with a phosphoprotein-specific gel stain. A total of 27 phosphoproteins were identified from 32 excised gel spots. The identified phosphoproteins indicate that LPS $_{\text {B.cep- }}$ induced signal perception/transduction involves G-proteincoupled receptor signaling, $\mathrm{Ca}^{2+} /$ calmodulin-dependent signaling pathways, $\mathrm{H}^{+}$-ATPase regulation of intracellular $\mathrm{pH}$, thioredoxin-mediated signaling, and phosphorylation of 14-3-3 regulatory proteins as well as phosphorylation of heat shock proteins, chaperones, and protein involved in biosynthesis and maintenance of nucleotide pools [38]. In order to obtain more information about the dynamic changes following perception of bacterial lipopolysaccharides, a multiplexed proteome analysis was also performed [39]. Proteomic and phosphoproteomic analyses identified 88 differentially regulated proteins and phosphoproteins that belong to five functional categories: proteins related to metabolism and energy-related processes (36), molecular chaperones and protein targeting (12), cell structure and cytoskeletal rearrangement proteins (8), proteins involved in RNA binding, protein synthesis and degradation (11), and cell transporters, ion homeostasis, and signal transductionrelated proteins (9). Notably, proteins with a role in the citric acid cycle, amino acid biosynthesis, sugar metabolism, and nucleotide synthesis were upregulated, as well as mitochondrial enzymes including ATP synthases. Molecular chaperones and proteins involved in protein synthesis and maturation such as Hsp60, calreticulin, luminal binding protein (BiP), and protein disulfide-isomerase were also upregulated as a result of LPS elicitation. This is consistent with the requirement for stabilization of proteins at intermediate stages of folding, assembly, vesicle trafficking, and secretion during the immune response. The abundance of cytoskeletal proteins also changed in response to LPS. Tubulins and actin showed increased phosphorylation [39], and this agrees with the dynamic reorganization of actin filaments and rapid depolymerization of microtubules observed in plantpathogen interactions [41]. In addition, LPS treatment led to an increase of vacuolar $\mathrm{H}^{+}$-ATPase subunits, and it has been reported that vacuolar $\mathrm{H}^{+}$-ATPase activity is required for endocytic secretory trafficking in Arabidopsis thaliana [42]. Proteosome components also displayed increased phosphorylation that may account for changes in protein stability or activation during the response. In the category of cell transporters, ion homeostasis and signal transduction, two porins from the outer mitochondrial membrane, showed increased phosphorylation, and this may suggest that increased transport of small molecules such as ATP and ADP across the mitochondrial outer membrane is an important response to LPS. In addition, two GTP-binding proteins showed increased abundance and a GTP-binding protein and a G-protein $\beta$ subunit-like protein displayed increased phosphorylation after LPS treatment, and this further supports the link between PAMP and signaling through G-protein-coupled receptors. The results emphasize the importance of the intricate interplay between reversible phosphorylation events, as catalyzed by protein kinases and phosphatases, and the eventual identification and analysis of the complete set of proteins differentially phosphorylated in response to LPS elicitation will provide new insights into defense signal transduction [39]. Many of the identi-

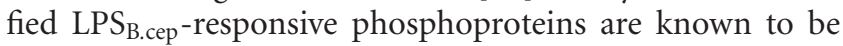
interconnected at many levels through a complex web of activation/deactivation, complex formation, protein-protein interactions, and chaperoning reactions. These studies thus provide novel insights and further evidence for the biochemical mechanism of LPS $_{\text {B.cep }}$ as a resistance elicitor, a PAMP, and triggering agent of defense responses associated with innate immunity $[38,39]$. 
With a view to better understand signaling events downstream of the PAMP molecules, the most interesting proteomics study was performed analyzing detergent-resistant membranes (DRMs) from A. thaliana suspension culture cells fractionated after the incubation with flagellin (flg22) [43]. The approach assumed that biological membranes form rafts in which proteins are associated with membranes in a dynamic manner and that differential stimuli can alter the raft proteome. Consequently, the most appropriate method to study these rafts was judged to be the isolation of DRMs [44]. The analysis of the Arabidopsis flg22responsive DRM proteome revealed 64 proteins that showed significant enrichment and characteristics such as transmembrane domains, glycosylphosphatidylinositol anchors, or lipid modifications that are diagnostic for membrane proteins. The enriched proteins include receptorlike kinases, and in particular the flagellin receptor FLS2, HERCULES1, and FERONIA, involved in cell elongation. The latter also has a role in signaling female control of pollen tube reception [45]. The identified proteins belonging to other categories include plasma membrane $\mathrm{H}^{+}$-ATPases, $\mathrm{Ca}^{2+}$-ATPases, vacuolar $\mathrm{H}^{+}$ATPases, different transporters, cell wall-related proteins, and proteins involved in cellular trafficking, metabolism, and stress/redox-related proteins. These results support the idea that flagellin treatment induces changes in plant plasma membrane compartmentalization [43].

Harpins are glycine-rich, cysteine-lacking, heat-stable proteins, secreted by the bacterial type three protein secretion system, that are able to trigger a hypersensitive response when infiltrated into nonhost plants [46]. Some harpins can bind to lipid bilayers and form ion-conducting pores [47], and it has been reported that they are secreted by the pathogen but not translocated into the plant cell [48], suggesting that they exert their function from the outside of the plant cell. This fact and the ability to elicit innate immune responses in plants in a MAPK-dependent manner $[49,50]$ led to proposing that harpins resemble PAMP molecules [6]. The effect of Pseudomonas syringae harpins on mitochondrial proteins of $A$. thaliana suspension culture cells was also analyzed by proteomics [51]. Twentyeight proteins were identified, and in combination with genome-wide transcriptional profiling, it was inferred that proteins from the citric acid cycle were upregulated pointing to an involvement of the mitochondria in the response to the harpin elicitor and a requirement for increased energy production during the defense response. Among the significantly upregulated proteins are the citrate synthase, $\mathrm{NAD}^{+}$isocitrate dehydrogenase, NAD-dependent malate dehydrogenase, the $\mathrm{E} 1 \beta$ subunit of pyruvate dehydrogenase, the ATP-dependent protease $\mathrm{ClpC} 2$, the actin depolimerizing factor ADF3, glutamine synthetase, and glutathione Stransferase.

In an attempt to evaluate the differences between PTI, disease, and ETI in the host-pathogen interaction model $A$. thaliana and Pseudomonas syringae pv. tomato, a proteomics analysis was undertaken. Leaves were either infected with wild type DC3000 (susceptibility), the bacteria expressing the effector protein AvrRpm1 (DC3000 (avrRpm1)) that elicits an ETI mediated by the cognate R-gene RPM1, or a $h r p$ mutant that elicits only basal responses. The analysis was restricted to total soluble protein, chloroplast-enriched, and mitochondria-enriched fractions [52]. Two antioxidant enzyme groups: the glutathione S-transferases (GSTs F2, F6, F7, and F8) and peroxiredoxins (PrxA, B, and IIE) showed differences in abundance after different times of bacteria infiltration implying that antioxidant enzymes play a critical role in modulating redox conditions of infected tissue with both pathogenic and nonpathogenic bacteria. Members of both enzyme groups showed multiple spots on gels that are consistent with specific post-translational modifications of these enzymes. Regarding the GST group, GSTF2, GSTF6, and GSTF7 changed in abundance after bacterial inoculations independently of the strain used, while GSTF8 was the only GST to show specificity for the R-gene response. Regarding the peroxiredoxins proteins, differential changes were observed with PrxIIE and to a lesser extent PrxB after bacteria challenge. PrxA did not show significant changes, however a lower molecular weight form of PrxA (PrxA-L) was reduced in response to wild type bacteria. This spot was further reduced when the plant was inoculated with the $h r p$ mutant, pointing to a targeted proteolysis of PrxA after the interaction with the pathogen and the possibility that this process is inhibited during the basal defense response. Taken together, it appears that Prxs and the antioxidants GSTs all respond to bacterial treatment and that specific posttranslational modifications in different proteins depend on the bacterial strain and the type of interaction. The authors also compared the protein and expression profiles for the corresponding GST and Prx genes by Affymetrix GeneChip analysis. In general, a good correlation was observed between proteins and transcript levels for the Prxs but not for the GST family that undergoes post-translational modifications. This therefore provides new insight into the role of early post-transcriptional modifications in the specific response against plant pathogens and underlines the importance of proteomics analyses in understanding host responses at the systems level.

In a further study $[53,54]$, a more complete proteomics analysis using the same experimental system could separate components of the basal defense (leaves inoculated with a mutant in the hypersensitive response and pathogenesis $(h r p)$ cluster) from disease and resistance responses following DC3000 and DC3000 (avrRpm1) inoculations. Moreover, since the proteomics of total soluble protein, chloroplastenriched, and mitochondria-enriched fractions were analyzed, the experimental design also provided insights into the spatial dynamics of the response. In this study, a total of 73 differential spots representing 52 unique proteins were successfully identified. The authors also correlated proteome changes with a transcriptomics analysis of the same response and observed that many of the changes in protein spot density occurred before significant transcriptional reprogramming between treatments was evident. Overall, the work showed that proteins that were subjected to significant changes after bacterial interactions were representative of two main functional groups, namely, defense-related antioxidants and metabolic enzymes. Components of the mitochondrial permeability transition such as mitochondrial porins 
appear to be modified early as part of the basal defense. General trends emerging from this broad study indicate that defense-related enzymes such as GSTs, peroxiredoxins and superoxide dismutase (SOD), and metabolic enzymes, including components of the citric acid cycle, rapidly alter their abundance during basal defense responses.

To date, experimental evidence suggests that PAMPinduced responses modify the host proteome and in particular proteins with a role in primary metabolism, redox modulation, molecular chaperones, cytoskeleton rearrangement, and signal transduction. In addition, the PAMP stimulus modifies mitochondrial and chloroplast proteome and reconfigures proteins in membrane rafts to enable efficient host signal transduction and downstream responses after the initial recognition.

\section{Proteomics of Host Susceptibility}

To colonize their hosts, bacteria need to coordinate population behavior, and this is achieved by the secretion of $\mathrm{N}$ acyl homoserine lactone (AHL) to enable "quorum sensing." Using proteomics, the changes in Medicago truncatula proteins exposed to bacterial AHLs from the pathogenic bacteria Pseudomonas aeruginosa were determined [55]. Comparisons of the response to the symbiotic bacteria Sinorhizobium meliloti revealed that the abundance of proteins depends on AHL structure, concentration, and time of exposure. Ninetynine proteins could be identified and based on homology were grouped into the following categories: energetics and various primary metabolic activities, plant defense or stress responses, protein degradation or processing, flavonoid synthesis, plant hormone responses or synthesis, regulatory functions, and cytoskeletal elements. Cytoskeletonassociated proteins such as actin-depolimerizing factor 2 and tubulin increased in abundance after $48 \mathrm{~h}$ of AHL treatment, and the Rubisco small subunit increased after $24 \mathrm{~h}$, reaffirming an involvement of cytoskeleton rearrangement and changing energy requirements during host interaction with the bacterial pathogen [55].

The interaction between Xanthomonas axonopodis pv. citri and citrus plants, that causes citrus canker, has also been examined by proteomics. The proteome modifications observed in citrus leaves infected with the pathogen include proteins related to carbon metabolism and photosynthesis, antioxidant proteins, and proteins related to stress responses and to plant protein trafficking. The most significant changes were observed in sugar-regulated photosynthetic proteins such as Rubisco and Rubisco activase as well as of ATP synthase that were downregulated, while NADH dehydrogenase was upregulated in infected leaves. Such a response is diagnostic for a reduction in photosynthetic efficiency during disease. Furthermore, an increase of proteins related to plant defense against the pathogen was found in $X$. axonopodis pv. citri treated leaves [56] affirming that de novo biosynthesis of defense-related compounds is essential for the host, while other cellular activities are reduced, thus permitting a reduction in photosynthetic rates until pathogenic growth has been slowed down or halted [57].
It has been proposed that such a reduction in photosynthesis may starve biotrophic pathogens of nutrients, thereby benefitting the host [58]. Surprisingly, X. axonopodis pv. citri encodes a plant-natriuretic-peptide- (PNP-) like gene (XacPNP) [59]. PNPs are a class of extracellular, systemically mobile peptides [60] that elicit a number of plant responses important in plant homeostasis and growth [61]. When XacPNP deletion mutants were infiltrated in citrus plants, lesions became more necrotic than those observed in the wild type infection, indicating that XacPNP is able to modulate host homeostasis to its own benefit $[62,63]$. Proteomic analyses were also performed on citrus leaves infected with $X$. axonopodis pv. citri lacking XacPNP and they showed a more pronounced decrease in photosynthetic proteins, while no reduction in defense-related proteins as compared to leaves infected with the wild type pathogen occurred. This indicated that XacPNP can serve the pathogen to prevent host photosynthetic shutdown during pathogenesis [56]. Further support for this hypothesis comes from the observation that infiltration of citrus leaves with recombinant XacPNP protein causes an increase in photosynthesis-related proteins [64]. In summary, the proteomics studies with wild type Xac, XacPNP deletion mutant, and recombinant protein support a role for the bacterial PNP in counteracting the shutdown of host photosynthesis during infection and the consequent maintenance of the host carbohydrate supply enabling prolonged biotrophic pathogen survival and colonization [58]. Again, proteome studies have afforded valuable insight into changes during the early onset of host-pathogen interactions that include cytoskeleton rearrangement and changes in the primary metabolism and that have far reaching consequences for the establishment of the disease. We also learn that the changes at the first stages are pronounced enough to leave a distinct proteomic footprint as part of the unfolding biotic stress response.

\section{Proteomics Comparison between Susceptibility and Resistance}

An interesting proteomics approach was undertaken to study the bacterial wilt of tomato caused by Ralstonia solanacearum that colonizes leaves, stems, and roots. The fact that roots from both resistant and susceptible tomato genotypes infected with $R$. solanacearum contained high numbers of pathogenic bacteria, while the population in the stem was significantly reduced in the resistant plant would suggest that differences in the proteomes might yield information about key components that underlie these observations. In an attempt to understand differences in host susceptibility, the protein patterns of the stems of susceptible and resistant plants were analyzed [65]. Firstly, the stem proteomes of the healthy plants of both genotypes showed no differences. Nevertheless, differentially regulated proteins in the susceptible genotype in response to $R$. solanacearum inoculation were understood to have a role in plant defense. They are STH-21, which is part of a small multigene family that is transcriptionally activated in potato upon infection [66] and TSI-1 (tomato stress induced-1), an intracellular PR protein 
and also part of a multigene family activated during the defense response [67]. While STH-21 was induced, TSI-1 was slightly downregulated upon infection. An enzyme involved in oxidative stress was also induced, a putative lactoylglutathione lyase which, in conjunction with glyoxalase I and II, is responsible for the detoxification of methylglioxal and other compounds formed as byproducts of carbohydrate and lipid metabolism [68]. Further, fructokinase and an ATP synthase $5 \beta$ subunit, proteins involved in carbohydrate metabolism and energy production, respectively, were induced upon infection. On the other hand, no proteins appeared differentially regulated in response to the bacterial pathogen in the resistant genotype, although the authors stated that the technique used (classical 2-D SDS-PAGE combined with Coomassie staining) has some limitations regarding detection of less abundant proteins and suggested that enrichment of fractionations and/or silver staining would increase the resolution [65].

Subsequently, a similar proteomics study expanded the analysis to subcellular fractions such as the stem cell wall proteome [69]. This approach was undertaken to simultaneously analyze the large spectrum of the stem cell wall protein associated both with susceptible and resistant tomato lines in the interaction with $R$. solanacearum. It was reported that in resistant plants, the spots corresponding to subtilase, peroxidase, BiP, fructokinase-2, nucleoside diphosphate kinase, and PII-like protein were more abundant as compared to mock inoculations. The spots annotated as BiP, stress-induced protein, catalase, enolase (2-phospho-D-glycerate hydrolase), vacuolar $\mathrm{H}^{+}$-ATPase, oxygen evolving protein (OEE) 2, eukaryotic translation initiation factor 5A (eTIF 5A)-3, and eTIF 5A-4 appear to be decreased. In turn, in the susceptible species, the increased spots were peroxidase, peroxidase cevil6, basic $30 \mathrm{kDa}$ endochitinase, triose phosphate isomerase, and a PR5 -like protein, while the decreased ones were $\alpha$-galactosidase ( $\alpha$-D -galactoside galactohydrolase), disulphide isomeraselike protein, xyloglucan endotransglucosylase-hydrolase7, two eTIF 5A-4, one eTIF 5A-1, and two glycine-rich proteins. The main differences observed between susceptible and resistant plants were BiP, three enolase, fructokinase-2, nascent polypeptide-associated complex (NAC)-a-like protein 3 , and OEE2 that are more abundant in the resistant species. The proteins that occurred at higher abundance in the susceptible species were $\alpha$-galactosidase, peroxidase, a hypothetical protein, ferredoxin-NADP reductase, OEE1, and eIF-5A-1. We may therefore conclude that both genotypes responded to Ralstonia by elevating the expression of PR and other defense related and glycolytic proteins, whereas in susceptible plants, cell wall and metabolic proteins appear downregulated, while in resistant lines, the same happens with antioxidant, stress-related, and energy metabolism proteins.

Since, beyond the cell wall, plasma membranes are the first point of contact with the pathogen, and the proteins localized therein play important roles in signal transduction; a recent study on plasma membrane proteins of Oryza sativa suspension cells challenged with two strains of the pathogen Xanthomonas oryzae pv. oryzae was undertaken with a view to elucidate the proteomes of the susceptible and resistant response. Differentially expressed pathogen-induced proteins included a vacuolar $\mathrm{H}^{+}$-ATPase $\beta$ subunit, protein phosphatases, quinone reductase, prohibitin (OsPHB2), hypersensitive induced response protein (OsHIR1), zinc finger, and $\mathrm{C} 2$ domain proteinlike, universal stress protein (USP), and a small heat shock protein, all of them are membrane proteins or membrane-associated proteins. Alcohol dehydrogenase 1 and ascorbate peroxidase were also identified in the assay since they may have been coisolated with the plasma membrane fraction. Most of these proteins showed similar patterns in response to both strains, and only a few showed discernible differential regulations. However, differences in protein abundance observed in the resistance model point to specific, albeit not yet well-understood roles of plasma membrane components in resistance responses [70].

Quantitative differences in the Arabidopsis proteome challenged with P. syringae pv. tomato DC3000 and DC3000 (avrRpm1) were also used to determine components of the resistance response [53, 54]. Some protein-folding and protein-binding components like 14-3-3 and cyclophillins changed in response to the TTSS, and many redox-related systems were also responsive to AvrRpm1/RPM1 interactions, including defense-related proteins such a GSTs. Significant changes in photosystem II (PSII) components were observed in the Rpm1-mediated resistance indicating that PSII may be a specific target of resistance signaling in response to AvrRpm1 [53, 54].

The modifications in the Arabidopsis response to the effector protein AvrRpm1 secreted by the pathogen $P$. syringae pv. tomato were further studied by proteome analysis of transgenic lines conditionally expressing avrRpm1 [71]. The Arabidopsis genome encodes a cognate R protein for Avr$\mathrm{Rpm} 1$ and another effector protein AvrB termed Rpm1. The protein phosphatase $2 \mathrm{C}$ renamed as PIA1 (PP2C induced by AvrRpm1) was detected in the transgenic line after fractionation and silver staining unmasking low-abundance proteins. PIA1 was also upregulated following infection with the bacteria, and its rapid appearance suggests a role in early Rpm-1, mediated signaling. No accumulation of PIA1 protein was seen following infection with $P$. syringae pv. tomato expressing AvrB. Although PIA1 transcripts did accumulate in response to avrB- or avrRpml-expressing strains; protein accumulation only occurred when AvrRpm1 was present. This result clearly highlights the value of a proteomics approach in elucidating mechanisms involved in defense signaling and responses to specific effectors in particular. In this case, PIAl has been discovered to be a protein marker that allows to distinguish AvrRpm1- from AvrB-dependent activation of RPM1 [71].

A further proteomics approach was conducted leading to a better understanding of the difference between the susceptible and the resistant response. The susceptible model was based on the interaction between $A$. thaliana and $P$. syringae pv. tomato DC3000, while in the resistant one, $A$. thaliana was challenged with the same bacteria expressing the effector protein AvrRpt2, DC3000 (AvrRpt2). In the resistant model, the abundance of several proteins involved 
in photosynthetic processes, including the large and small subunits of Rubisco and Rubisco activase, was reduced, while a number of proteins associated with host defense were accumulated. These included redox-related proteins such as oxidoreductase, quinone reductase, and a massive production of glutathione peroxidase as well as several other defense-related proteins. Overall, these changes reflect a switch from primary metabolism to defense metabolism. On the other hand, the virulent infection did not appear to affect the stress metabolism, and only a few redox-related proteins seem differentially regulated. Furthermore, several of the accumulated proteins function in protein folding, for example, peptidyl-prolyl cis-trans isomerase. In the same series of experiments, an A. thaliana $S$-nitrosoglutathione reductase mutant, impaired in nitric oxide (NO) signaling, was used to demonstrate the importance of $\mathrm{NO}$ in plant defense responses at the proteomics level. Although the protein pattern obtained showed overlapping defense responses with wild type plants, in the NO altered signaling mutant; the most striking difference is the lack of change of photosynthetic process proteins and a reduction in stress-related and redox-related proteins relative to the wild type. These results therefore further affirm that proteomics is the most valuable tool for the evaluation of the complex mechanisms involved in host defense responses [72].

Bacterial canker of tomato is caused by the gram-positive bacterium Clavibacter michiganensis subsp. michiganensis and is a devastating disease that proliferates in the xylem vessels of infected plants. However, several wild tomato species possess resistance to this pathogen, in particular accessions of the species Lycopersicon hirsutum and $L$. peruvianum. In order to identify proteins expressed in response to $C$. michiganensis, a proteomics experiment was conducted comparing protein profiles of introgression lines containing two quantitative trait loci (QTL), Rcm 2.0, and Rcm 5.1 from L. hirsutum accession LA407 with a susceptible control line [73]. These loci control partial resistance to genetically diverse $C$. michiganensis subsp. michiganensis strains. The three lines tested share $93.75 \%$ of their genome with L. esculentum, which is a susceptible species. The protein pattern was evaluated at 72 and $144 \mathrm{~h}$ post-inoculation (hpi) with the pathogenic bacteria and the accumulation of specific proteins was dependent on the genotype and time post-inoculation. Three distinct SOD enzymes were differentially expressed among genotypes in response to $C$. michiganensis subsp. michiganensis implying that oxidative stress is a part of the response to infection. In addition to the SOD enzymes, a number of proteins involved in defense and stress responses were differentially expressed in the different genotypes. In genotypes, $\mathrm{Rcm}$ 2.0, remorin, phospholipid glutathione peroxidase, and PR-3 were upregulated. Remorin is present as a single-copy gene in L. esculentum and is involved in cell-to-cell signaling, molecular transport, and the disease and wound response [74]. Remorin and phospholipid glutathione peroxidase were detected in inoculated plants of the genotype $\mathrm{Rcm} 2.0$ at $72 \mathrm{hpi}$ and in all inoculated genotypes at $144 \mathrm{hpi}$, although the induction was marginal for phospholipid glutathione peroxidase. The pathogenesis-related protein PR-3 was differentially upregulated in resistant genotypes Rcm 2.0 at 144 hpi. Therefore, lines containing Rcm 2.0 responded uniquely to the presence of $C$. michiganensis subsp. michiganensis, probably because they are able to respond earlier to infection. An alcohol dehydrogenase was uniquely upregulated in Rcm 5.1 genotype during infection. Two additional defenserelated proteins, a GST and a carbonic anhydrase, were found to be upregulated in both $\mathrm{Rcm} 5.1$ genotypes and control line after pathogen inoculation. Differential expression of regulatory proteins was also detected in response to pathogen inoculation. A protein translation inhibitor, RNA-binding protein, and $S$-adenosylhomocysteine hydrolase were upregulated in Rcm 5.1 genotype. Several proteins involved in carbon metabolism were reported to be downregulated in lines containing Rcm 2.0 and Rcm 5.1. Ribulose-phosphate 3epimerase, Rubisco activase, triose phosphate isomerase, and sedoheptulose-1,7-bisphosphatase were all downregulated in resistant lines [73].

Proteomics was also applied to compare differences between compatible and incompatible interactions mediated by an $\mathrm{R}$ protein in rice [75]. Proteins were obtained from leaves of Rice cv. Java 14 seedlings inoculated with compatible (Xo7435) and incompatible (T7174) races of X. oryzae pv. Oryzae, and 20 differentially expressed proteins were identified in response to bacterial inoculation. These proteins were categorized into classes relating to energy, metabolism, and defense. Among the 20 proteins, the Rubisco large subunit was fragmented into two smaller proteins in inoculations with both pathogens. Protein changes after the treatment with jasmonic acid revealed that it mediated changes in abundance of five of the identified proteins. In particular, a thaumatinlike protein and probenazole-inducible protein (PBZ) were commonly upregulated by both bacterial inoculations as well as JA treatment confirming that JA-dependent processes are involved in the defense of rice against bacterial blight caused by bacteria $X$. oryzae pv. oryzae [75].

Taken together, these proteomics-based investigations are consistent with the view that susceptible and resistant plants have biochemically distinct response signatures and that some of these signatures are clearly diagnostic for specific responses. Pathogenesis-related proteins, glycolytic proteins, specific plasma membrane and plant cell wall proteins, antioxidant proteins and redox-related proteins, some protein-folding or protein-binding components, stress-related proteins, and energy metabolism proteins are differentially regulated in susceptible and resistant plants. SOD and GST regulations in the resistance to tomato bacterial canker [73] and the protein phosphatase $2 \mathrm{C}$ in the Arabidopsis response to $P$. syringae pv. tomato are examples of differentially regulated proteins [71]. We also note that PSII may be a component of the host response highlighting the importance of PSII in Rpm1-mediated resistance [53]. Further to the differential expression directly observed, the results obtained in these studies support the hypothesis that resistance mechanisms involve complex multifaceted interactions of proteins where their specific modulation and activation kinetics may play important roles that await further molecular analyses. 


\section{Proteomics as Part of the Systems Analyses in Model Species}

The use of model systems has both significant benefits and limitations. In the case of $A$. thaliana, the benefits include the availability of the complete genome, many well-defined mutants, large repositories of transcription data in both wild type and mutants in the public domain, and many publicly available computational analysis tools [76]. These tools help to infer gene function based on coexpression studies and enrichments in ontological categories of positively and negatively expressed correlated genes [76, 77]. The availability of the complete genome also enables computational analyses of the promoter sequences of expression-correlated genes which in turn allow identification of enriched-transcriptionfactor-binding sites. This type of sequential computational analysis has been applied previously to support the interpretation of the data obtained in proteomics studies [64]. In this case, differentially expressed proteins in response to a signaling peptide in citrus were first identified with proteomics. Subsequently, the orthologs in Arabidopsis were identified and used for the computational analysis in the model organism. The expanded analyses will allow us to infer functions of individual proteins based on the principle of "guilt by association" and provide clues on the temporal, spatial, and stimulus-specific nature of the responses. In fact, the value of any proteomics result is enhanced if it is considered together with transcriptomic results since it is precisely the differences in these responses that are diagnostic for responses that cannot be properly understood by looking at the levels of proteins or RNAs only.

Finally, given that signaling and/or activation through (auto)phosphorylation is fundamental to cellular function, and intracellular communication in particular, phosphoproteomics has been developed and applied to elucidate plant responses $[38,78,79]$. The aim here is to resolve temporal and stimulus-specific phosphorylation events with a view to resolve global and quantitative changes in the phosphorylation status of individual proteins in the proteome. We can expect to gain most valuable insights from phosphoproteomics, particularly into the "crosstalk" between different stimuli and hormones. Here again, the use of the Arabidopsis model system will prove highly valuable since we have access to many hormone deficient and/or insensitive mutants, mutants that constitutively express defense programs or lack key components of the defense program.

\section{Concluding Remarks}

Plant-bacteria interactions are highly complex since multiple bacterial factors and plant-signaling events take place, which ultimately define the susceptibility or resistance of the plant exposed to the pathogen. Several recent and current investigations are directed towards gaining a better understanding of the molecular mechanisms implicated in basal and specific plant defense responses against plant bacterial pathogens. This review summarizes the use of proteomic approaches and insights from them that eventually will significantly impact our understanding of the molecular and cellular processes that govern host responses such as PTI, ETS, and ETI. Additionally, the detailed global comparison of response pathways using proteomics has allowed the identification of novel proteins whose biological role warrants in-depth biochemical and cellular elucidation. Finally, a systemlevel understanding of biotic stress responses may identify promising novel targets for the development of cultivars with improved disease resistance.

\section{Acknowledgment}

This work was supported by grants from Argentine Federal Government (ANPCyT PICT2010-1507 to N. Gottig, PICT2010-0300 to J. Ottado and PIP 2010-2012 to N. Gottig and J. Ottado). N. Gottig and J. Ottado are staff members and T. Zimaro and B. S. Garavaglia are fellows of the Consejo Nacional de Investigaciones Científicas y Técnicas (CONICET, Argentina).

\section{References}

[1] J. D. Jones and J. L. Dangl, "The plant immune system," Nature, vol. 444, no. 7117, pp. 323-329, 2006.

[2] G. Felix, J. D. Duran, S. Volko, and T. Boller, "Plants have a sensitive perception system for the most conserved domain of bacterial flagellin," The Plant Journal, vol. 18, no. 3, pp. 265276, 1999.

[3] M. Dow, M. A. Newman, and E. von Roepenack, "The induction and modulation of plant defense responses by bacterial lipopolysaccharides," Annual Review of Phytopathology, vol. 38, pp. 241-261, 2000.

[4] A. A. Gust, R. Biswas, H. D. Lenz et al., "Bacteria-derived peptidoglycans constitute pathogen-associated molecular patterns triggering innate immunity in Arabidopsis," Journal of Biological Chemistry, vol. 282, no. 44, pp. 32338-32348, 2007.

[5] G. Erbs, A. Molinaro, J. M. Dow, and M. A. Newman, "Lipopolysaccharides and plant innate immunity," SubCellular Biochemistry, vol. 53, pp. 387-403, 2010.

[6] S. Engelhardt, J. Lee, Y. Gäbler et al., "Separable roles of the Pseudomonas syringae pv. phaseolicola accessory protein HrpZ1 in ion-conducting pore formation and activation of plant immunity," The Plant Journal, vol. 57, no. 4, pp. 706$717,2009$.

[7] W. Ma, K. Yoshioka, C. Gehring, and G. Berkowitz, "The function of cyclic nucleotide-gated channels in biotic stress," in Ion Channels and Plant Stress Responses, Signaling and Communication in Plants, V. Demidchik and F. Maathuis, Eds., pp. 159-174, Springer, Berlin, Germany, 2010.

[8] Z. Qia, R. Verma, C. Gehring et al., " $\mathrm{Ca}^{2+}$ signaling by plant Arabidopsis thaliana Pep peptides depends on AtPepR1, a receptor with guanylyl cyclase activity, and cGMP-activated $\mathrm{Ca}^{2+}$ channels," Proceedings of the National Academy of Sciences of the United States of America, vol. 107, no. 49, pp. 2119321198, 2010.

[9] S. T. Chisholm, G. Coaker, B. Day, and B. J. Staskawicz, "Host-microbe interactions: shaping the evolution of the plant immune response," Cell, vol. 124, no. 4, pp. 803-814, 2006.

[10] J. D. Lewis, D. S. Guttman, and D. Desveaux, "The targeting of plant cellular systems by injected type III effector proteins," Seminars in Cell and Developmental Biology, vol. 20, no. 9, pp. 1055-1063, 2009. 
[11] P. Hauck, R. Thilmony, and S. Y. He, "A Pseudomonas syringae type III effector suppresses cell wall-based extracellular defense in susceptible Arabidopsis plants," Proceedings of the National Academy of Sciences of the United States of America, vol. 100, no. 14, pp. 8577-8582, 2003.

[12] M. G. Kim, L. da Cunha, A. J. McFall et al., "Two Pseudomonas syringae type III effectors inhibit RIN4-regulated basal defense in Arabidopsis," Cell, vol. 121, no. 5, pp. 749-759, 2005.

[13] W. Zhu, B. Yang, J. M. Chittoor, L. B. Johnson, and F. F. White, "AvrXa10 contains an acidic transcriptional activation domain in the functionally conserved C terminus," Molecular PlantMicrobe Interactions, vol. 11, no. 8, pp. 824-832, 1998.

[14] B. Yang, W. Zhu, L. B. Johnson, and F. F. White, "The virulence factor AvrXa7 of Xanthomonas oryzae pv. oryzae is a type III secretion pathway-dependent nuclear-localized doublestranded DNA-binding protein," Proceedings of the National Academy of Sciences of the United States of America, vol. 97, no. 17, pp. 9807-9812, 2000.

[15] P. He, S. Chintamanani, Z. Chen et al., "Activation of a COI1dependent pathway in Arabidopsis by Pseudomonas syringae type III effectors and coronatine," The Plant Journal, vol. 37, no. 4, pp. 589-602, 2004.

[16] J. L. Dangl and J. D. Jones, "Plant pathogens and integrated defence responses to infection," Nature, vol. 411, no. 6839, pp. 826-833, 2001.

[17] B. P. Thomma, T. Nürnberger, and M. H. Joosten, "Of PAMPs and effectors: the blurred PTI-ETI dichotomy," The Plant Cell, vol. 23, no. 1, pp. 4-15, 2011.

[18] J. Lee, B. Klüsener, G. Tsiamis et al., "HrpZPsph from the plant pathogen Pseudomonas syringae pv. phaseolicola binds to lipid bilayers and forms an ion-conducting pore in vitro," Proceedings of the National Academy of Sciences of the United States of America, vol. 98, no. 1, pp. 289-294, 2001.

[19] G. Felix and T. Boller, "Molecular sensing of bacteria in plants: the highly conserved RNA-binding motif RNP-1 of bacterial cold shock proteins is recognized as an elicitor signal in tobacco," Journal of Biological Chemistry, vol. 278, no. 8, pp. 6201-6208, 2003.

[20] C. Zipfel, G. Kunze, D. Chinchilla et al., "Perception of the bacterial PAMP EF-Tu by the receptor EFR restricts Agrobacterium-mediated transformation," Cell, vol. 125, no. 4, pp. 749-760, 2006.

[21] P. N. Dodds and J. P. Rathjen, "Plant immunity: towards an integrated view of plant-pathogen interactions," Nature Reviews Genetics, vol. 11, no. 8, pp. 539-548, 2010.

[22] T. E. Mishina and J. Zeier, "Pathogen-associated molecular pattern recognition rather than development of tissue necrosis contributes to bacterial induction of systemic acquired resistance in Arabidopsis," The Plant Journal, vol. 50, no. 3, pp. 500-513, 2007.

[23] L. Wirthmueller, Y. Zhang, J. D. G. Jones, and J. E. Parker, "Nuclear accumulation of the Arabidopsis immune receptor RPS4 is necessary for triggering EDS1-dependent defense," Current Biology, vol. 17, no. 23, pp. 2023-2029, 2007.

[24] L. Navarro, C. Zipfel, O. Rowland et al., "The transcriptional innate immune response to flg22. Interplay and overlap with Avr gene-dependent defense responses and bacterial pathogenesis," Plant Physiology, vol. 135, no. 2, pp. 1113-1128, 2004.

[25] R. A. Cernadas, L. R. Camillo, and C. E. Benedetti, "Transcriptional analysis of the sweet orange interaction with the citrus canker pathogens Xanthomonas axonopodis pv. citri and Xanthomonas axonopodis pv. aurantifolii," Molecular Plant Pathology, vol. 9, no. 5, pp. 609-631, 2008.
[26] W. Truman, M. T. De Zabala, and M. Grant, "Type III effectors orchestrate a complex interplay between transcriptional networks to modify basal defence responses during pathogenesis and resistance," The Plant Journal, vol. 46, no. 1, pp. 14-33, 2006.

[27] Z. Cheng, O. Woody, B. Glick, and B. McConkey, "Characterization of plant-bacterial interactions using proteomic approaches," Current Proteomics, vol. 7, no. 4, pp. 244-257, 2010.

[28] A. Mehta, A. C. Brasileiro, D. S. Souza et al., "Plant-pathogen interactions: what is proteomics telling us?" FEBS Journal, vol. 275 , no. 15 , pp. 3731-3746, 2008.

[29] U. Mathesius, "Comparative proteomic studies of rootmicrobe interactions," Journal of Proteomics, vol. 72, no. 3, pp. 353-366, 2009.

[30] I. Miller, J. Crawford, and E. Gianazza, "Protein stains for proteomic applications: which, when, why?" Proteomics, vol. 6, no. 20, pp. 5385-5408, 2006.

[31] R. Westermeier and R. Marouga, "Protein detection methods in proteomics research," Bioscience Reports, vol. 25, no. 1-2, pp. 19-32, 2005.

[32] W. F. Patton, "Detection technologies in proteome analysis," Journal of Chromatography B, vol. 771, no. 1-2, pp. 3-31, 2002.

[33] M. Hamdan and P. G. Righetti, "Modern strategies for protein quantification in proteome analysis: advantages and limitations," Mass Spectrometry Reviews, vol. 21, no. 4, pp. 287-302, 2002.

[34] M. Ünlü, M. E. Morgan, and J. S. Minden, "Difference gel electrophoresis: a single gel method for detecting changes in protein extracts," Electrophoresis, vol. 18, no. 11, pp. 20712077, 1997.

[35] P. A. Haynes and T. H. Roberts, "Subcellular shotgun proteomics in plants: looking beyond the usual suspects," Proteomics, vol. 7, no. 16, pp. 2963-2975, 2007.

[36] A. Pirondini, G. Visioli, A. Malcevschi, and N. Marmiroli, "A 2-D liquid-phase chromatography for proteomic analysis in plant tissues," Journal of Chromatography B, vol. 833, no. 1, pp. 91-100, 2006.

[37] M. Ye, X. Jiang, S. Feng, R. Tian, and H. Zou, "Advances in chromatographic techniques and methods in shotgun proteome analysis," Trends in Analytical Chemistry, vol. 26, no. 1, pp. 80-84, 2007.

[38] I. B. Gerber, K. Laukens, E. Witters, and I. A. Dubery, "Lipopolysaccharide-responsive phosphoproteins in Nicotiana tabacum cells," Plant Physiology and Biochemistry, vol. 44, no. 5-6, pp. 369-379, 2006.

[39] I. B. Gerber, K. Laukens, T. De Vijlder, E. Witters, and I. A. Dubery, "Proteomic profiling of cellular targets of lipopolysaccharide-induced signalling in Nicotiana tabacum BY-2 cells," Biochimica et Biophysica Acta, vol. 1784, no. 11, pp. 1750-1762, 2008.

[40] H. Coventry and I. Dubery, "Lipopolysaccharides from Burkholderia cepacia contribute to an enhanced defensive capacity and the induction of pathogenesis-related proteins in Nicotianae tabacum," Physiological and Molecular Plant Pathology, vol. 58, no. 4, pp. 149-158, 2001.

[41] B. Day, J. L. Henty, K. J. Porter, and C. J. Staiger, "The pathogen-actin connection: a platform for defense signaling in plants," Annual Review of Phytopathology, vol. 49, pp. 483506, 2011.

[42] J. Dettmer, A. Hong-Hermesdorf, Y. D. Stierhof, and K. Schumacher, "Vacuolar $\mathrm{H}^{+}$-ATPase activity is required for endocytic and secretory trafficking in Arabidopsis," The Plant Cell, vol. 18, no. 3, pp. 715-730, 2006. 
[43] N. F. Keinath, S. Kierszniowska, J. Lorek et al., "PAMP (Pathogen-associated Molecular Pattern)-induced changes in plasma membrane compartmentalization reveal novel components of plant immunity," Journal of Biological Chemistry, vol. 285, no. 50, pp. 39140-39149, 2010.

[44] L. J. Foster, C. L. De Hoog, and M. Mann, "Unbiased quantitative proteomics of lipid rafts reveals high specificity for signaling factors," Proceedings of the National Academy of Sciences of the United States of America, vol. 100, no. 10, pp. 5813-5818, 2003.

[45] H. Guo, L. Li, H. Ye, X. Yu, A. Algreen, and Y. Yin, "Three related receptor-like kinases are required for optimal cell elongation in Arabidopsis thaliana," Proceedings of the National Academy of Sciences of the United States of America, vol. 106, no. 18, pp. 7648-7653, 2009.

[46] S. Y. He, H. C. Huang, and A. Collmer, "Pseudomonas syringae pv. syringae $\operatorname{Harpin}_{P s s}$ : a protein that is secreted via the Hrp pathway and elicits the hypersensitive response in plants," Cell, vol. 73, no. 7, pp. 1255-1266, 1993.

[47] M. Haapalainen, S. Engelhardt, I. Küfner et al., "Functional mapping of harpin HrpZ of Pseudomonas syringae reveals the sites responsible for protein oligomerization, lipid interactions and plant defence induction," Molecular Plant Pathology, vol. 12, no. 2, pp. 151-166, 2011.

[48] D. Büttner, D. Gürlebeck, L. D. Noël, and U. Bonas, "HpaB from Xanthomonas campestris pv. vesicatoria acts as an exit control protein in type III-dependent protein secretion," Molecular Microbiology, vol. 54, no. 3, pp. 755-768, 2004.

[49] J. Lee, D. F. Klessig, and T. Nürnberger, "A harpin binding site in tobacco plasma membranes mediates activation of the pathogenesis-related gene HIN1 independent of extracellular calcium but dependent on mitogen-activated protein kinase activity," Plant Cell, vol. 13, no. 5, pp. 1079-1093, 2001.

[50] R. Desikan, J. T. Hancock, K. Ichimura, K. Shinozaki, and S. J. Neill, "Harpin induces activation of the arabidopsis mitogen-activated protein kinases AtMPK4 and AtMPK6," Plant Physiology, vol. 126, no. 4, pp. 1579-1587, 2001.

[51] M. Livaja, M. C. Palmieri, U. von Rad, and J. Durner, "The effect of the bacterial effector protein harpin on transcriptional profile and mitochondrial proteins of Arabidopsis thaliana," Journal of Proteomics, vol. 71, no. 2, pp. 148-159, 2008.

[52] A. M. Jones, V. Thomas, B. Truman, K. Lilley, J. Mansfield, and M. Grant, "Specific changes in the Arabidopsis proteome in response to bacterial challenge: differentiating basal and Rgene mediated resistance," Phytochemistry, vol. 65, no. 12, pp. 1805-1816, 2004.

[53] A. M. Jones, V. Thomas, M. H. Bennett, J. Mansfield, and M. Grant, "Modifications to the arabidopsis defense proteome occur prior to significant transcriptional change in response to inoculation with Pseudomonas syringae," Plant Physiology, vol. 142, no. 4, pp. 1603-1620, 2006.

[54] A. M. Jones, J. Mansfield, and M. Grant, "Considerations on post-translational modification and protein targeting in the arabidopsis defense proteome," Plant Signaling and Behavior, vol. 2, no. 3, pp. 153-154, 2007.

[55] U. Mathesius, S. Mulders, M. Gao et al., "Extensive and specific responses of a eukaryote to bacterial quorum-sensing signals," Proceedings of the National Academy of Sciences of the United States of America, vol. 100, no. 3, pp. 1444-1449, 2003.

[56] B. S. Garavaglia, L. Thomas, N. Gottig et al., "A eukaryoticacquired gene by a biotrophic phytopathogen allows prolonged survival on the host by counteracting the shut-down of plant photosynthesis," PloS One, vol. 5, no. 1, p. e8950, 2010.
[57] M. D. Bolton, "Primary metabolism and plant defense-fuel for the fire," Molecular Plant-Microbe Interactions, vol. 22, no. 5, pp. 487-497, 2009.

[58] B. S. Garavaglia, L. Thomas, N. Gottig et al., "Shedding light on the role of photosynthesis in pathogen colonization and host defense," Communicative and Integrative Biology, vol. 3, no. 4, pp. 382-384, 2010.

[59] V. Nembaware, C. Seoighe, M. Sayed, and C. Gehring, "A plant natriuretic peptide-like gene in the bacterial pathogen Xanthomonas axonopodis may induce hyper-hydration in the plant host: a hypothesis of molecular mimicry," $B M C$ Evolutionary Biology, vol. 4, article 10, 2004.

[60] Y. H. Wang, C. Gehring, and H. R. Irving, "Plant natriuretic peptides are apoplastic and paracrine stress response molecules," Plant and Cell Physiology, vol. 52, no. 5, pp. 837850, 2011.

[61] C. A. Gehring and H. R. Irving, "Natriuretic peptides-a class of heterologous molecules in plants," The International Journal of Biochemistry and Cell Biology, vol. 35, no. 9, pp. 1318-1322, 2003.

[62] N. Gottig, B. S. Garavaglia, L. D. Daurelio et al., "Xanthomonas axonopodis pv. citri uses a plant natriuretic peptide-like protein to modify host homeostasis," Proceedings of the National Academy of Sciences of the United States of America, vol. 105, no. 47, pp. 18631-18636, 2008.

[63] N. Gottig, B. S. Garavaglia, L. D. Daurelio et al., "Modulating host homeostasis as a strategy in the plant-pathogen arms race," Communitative and Integrative Biology, vol. 2, no. 2, pp. 89-90, 2009.

[64] B. S. Garavaglia, L. Thomas, T. Zimaro et al., "A plant natriuretic peptide-like molecule of the pathogen Xanthomonas axonopodis pv. citri causes rapid changes in the proteome of its citrus host," BMC plant biology, vol. 10, p. 51, 2010.

[65] D. Dahal, D. Heintz, A. Van Dorsselaer, H. P. Braun, and K. Wydra, "Pathogenesis and stress related, as well as metabolic proteins are regulated in tomato stems infected with Ralstonia solanacearum," Plant Physiology and Biochemistry, vol. 47, no. 9, pp. 838-846, 2009.

[66] C. P. Constabel, C. Bertrand, and N. Brisson, "Transgenic potato plants overexpressing the pathogenesis-related STH-2 gene show unaltered susceptibility to Phytophthora infestans and potato virus X," Plant Molecular Biology, vol. 22, no. 5, pp. 775-782, 1993.

[67] C. Sree Vidya, M. Manoharan, and S. Lakshmi, "Cloning and characterization of salicylic acid-induced, intracellular pathogenesis-related gene from tomato (Lycopersicon esculentum)," Journal of Biosciences, vol. 24, no. 3, pp. 287-293, 1999.

[68] S. L. Singla-Pareek, M. K. Reddy, and S. K. Sopory, "Genetic engineering of the glyoxalase pathway in tobacco leads to enhanced salinity tolerance," Proceedings of the National Academy of Sciences of the United States of America, vol. 100, no. 25, pp. 14672-14677, 2003.

[69] D. Dahal, A. Pich, H. P. Braun, and K. Wydra, "Analysis of cell wall proteins regulated in stem of susceptible and resistant tomato species after inoculation with Ralstonia solanacearum: a proteomic approach," Plant Molecular Biology, vol. 73, no. 6, pp. 643-658, 2010.

[70] F. Chen, Y. Yuan, Q. Li, and Z. He, "Proteomic analysis of rice plasma membrane reveals proteins involved in early defense response to bacterial blight," Proteomics, vol. 7, no. 9, pp. 1529-1539, 2007.

[71] I. Widjaja, I. Lassowskat, G. Bethke et al., "A protein phosphatase 2C, responsive to the bacterial effector AvrRpm1 but not to the AvrB effector, regulates defense responses in 
Arabidopsis," The Plant Journal, vol. 61, no. 2, pp. 249-258, 2010.

[72] C. Holzmeister, A. Fröhlich, H. Sarioglu, N. Bauer, J. Durner, and C. Lindermayr, "Proteomic analysis of defense response of wildtype Arabidopsis thaliana and plants with impaired NOhomeostasis," Proteomics, vol. 11, no. 9, pp. 1664-1683, 2011.

[73] G. L. Coaker, B. Willard, M. Kinter, E. J. Stockinger, and D. M. Francis, "Proteomic analysis of resistance mediated by $\mathrm{Rcm} 2.0$ and $\mathrm{Rcm}$ 5.1, two loci controlling resistance to bacterial canker of tomato," Molecular Plant-Microbe Interactions, vol. 17, no. 9, pp. 1019-1028, 2004.

[74] P. Reymond, B. Kunz, K. Paul-Pletzer, R. Grimm, C. Eckerskorn, and E. E. Farmer, "Physiologists cloning of a cDNA encoding a plasma membrane-associated, uronide binding phosphoprotein with physical properties similar to viral movement proteins," The Plant Cell, vol. 8, no. 12, pp. 22652276, 1996.

[75] T. Mahmood, A. Jan, M. Kakishima, and S. Komatsu, "Proteomic analysis of bacterial-blight defense-responsive proteins in rice leaf blades," Proteomics, vol. 6, no. 22, pp. 6053-6065, 2006.

[76] S. Meier and C. Gehring, "A guide to the integrated application of on-line data mining tools for the inference of gene functions at the systems level," Biotechnology Journal, vol. 3, no. 11, pp. 1375-1387, 2008.

[77] S. Meier, R. Bastian, L. Donaldson, S. Murray, V. Bajic, and C. Gehring, "Co-expression and promoter content analyses assign a role in biotic and abiotic stress responses to plant natriuretic peptides," BMC Plant Biology, vol. 8, article 24, 2008.

[78] Y. Chen, W. Hoehenwarter, and W. Weckwerth, "Comparative analysis of phytohormone-responsive phosphoproteins in Arabidopsis thaliana using $\mathrm{TiO}_{2}$-phosphopeptide enrichment and mass accuracy precursor alignment," The Plant Journal, vol. 63, no. 1, pp. 1-17, 2010.

[79] K. G. Kline, G. A. Barrett-Wilt, and M. R. Sussman, "In planta changes in protein phosphorylation induced by the plant hormone abscisic acid," Proceedings of the National Academy of Sciences of the United States of America, vol. 107, no. 36, pp. 15986-15991, 2010. 

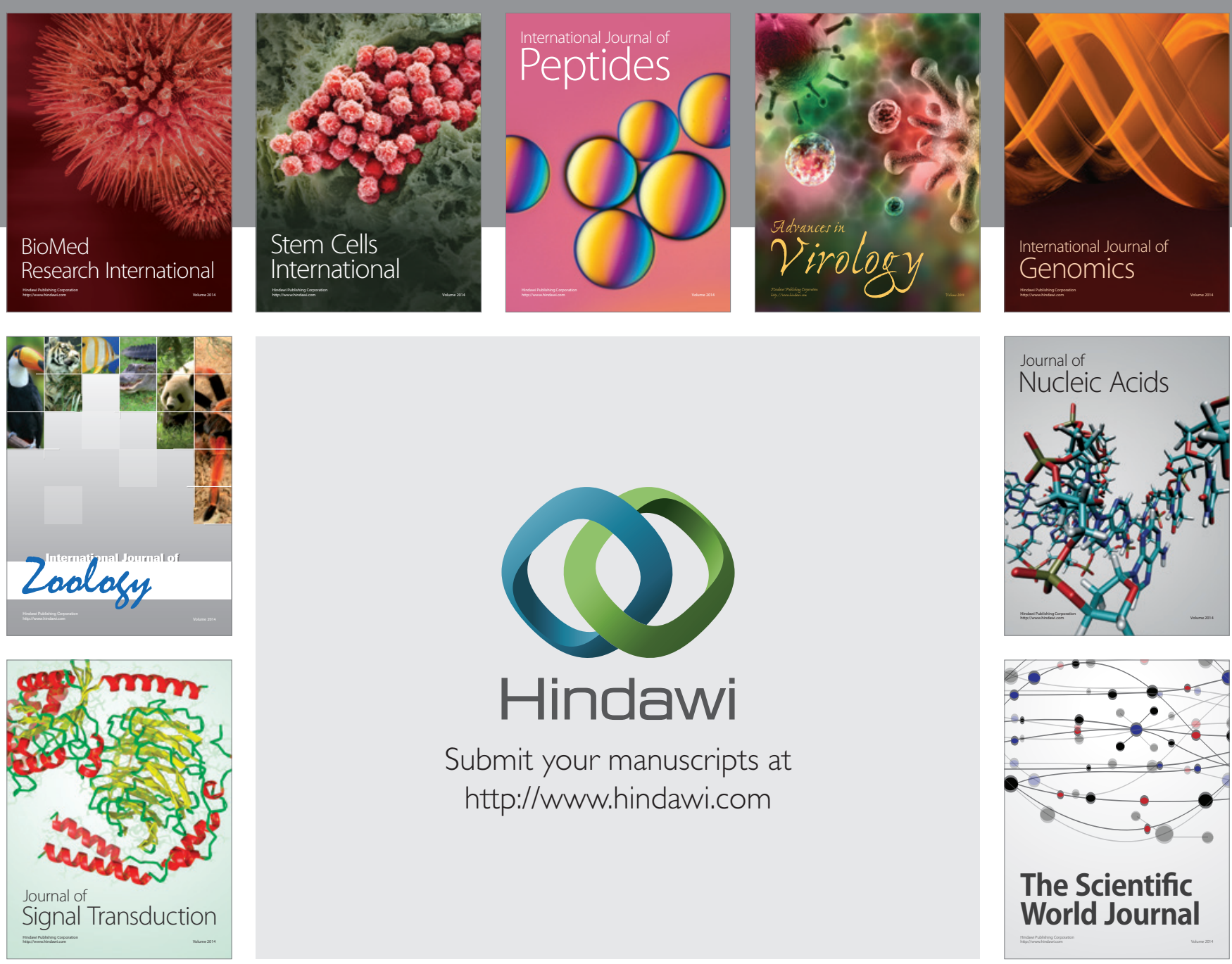

Submit your manuscripts at

http://www.hindawi.com
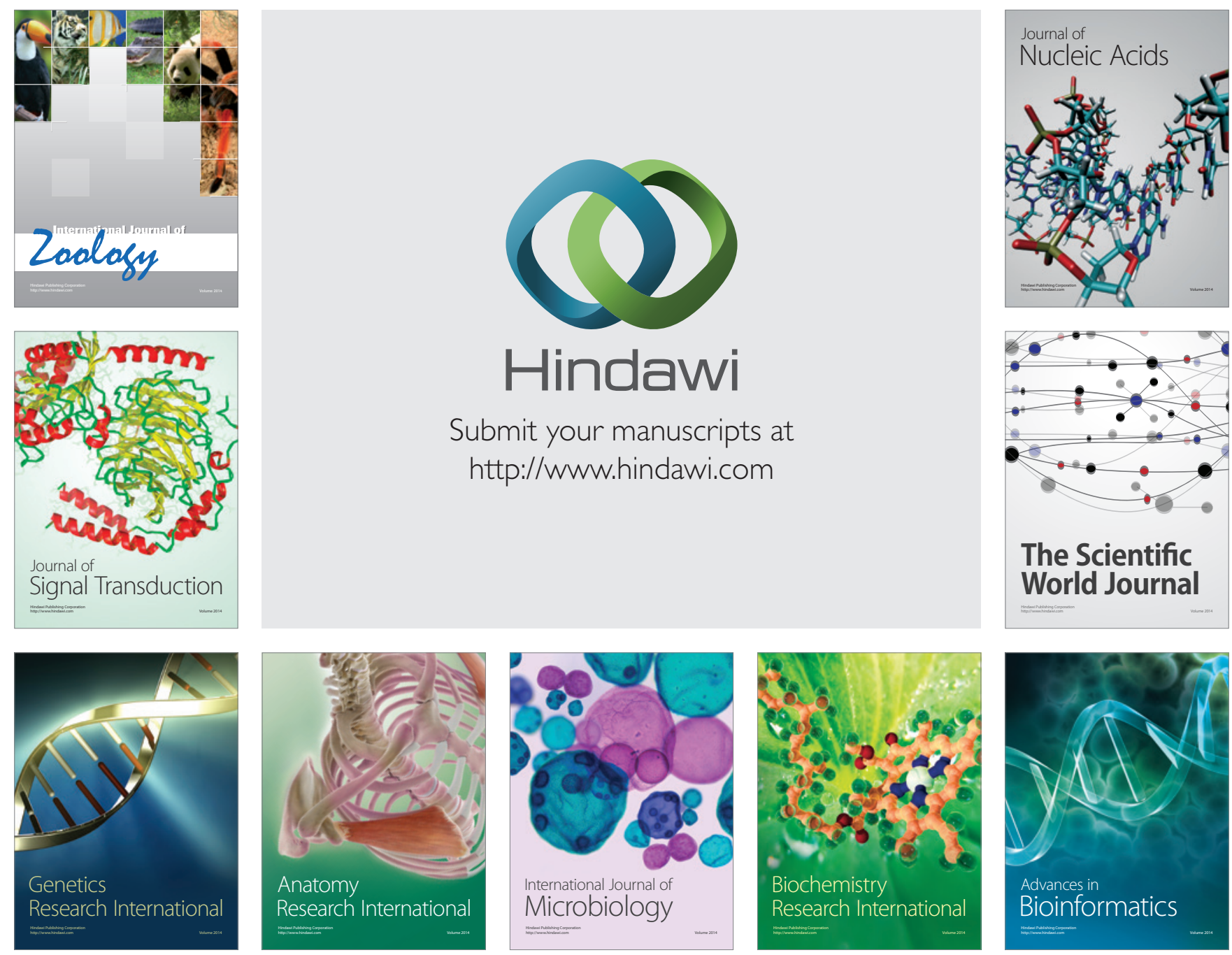

The Scientific World Journal
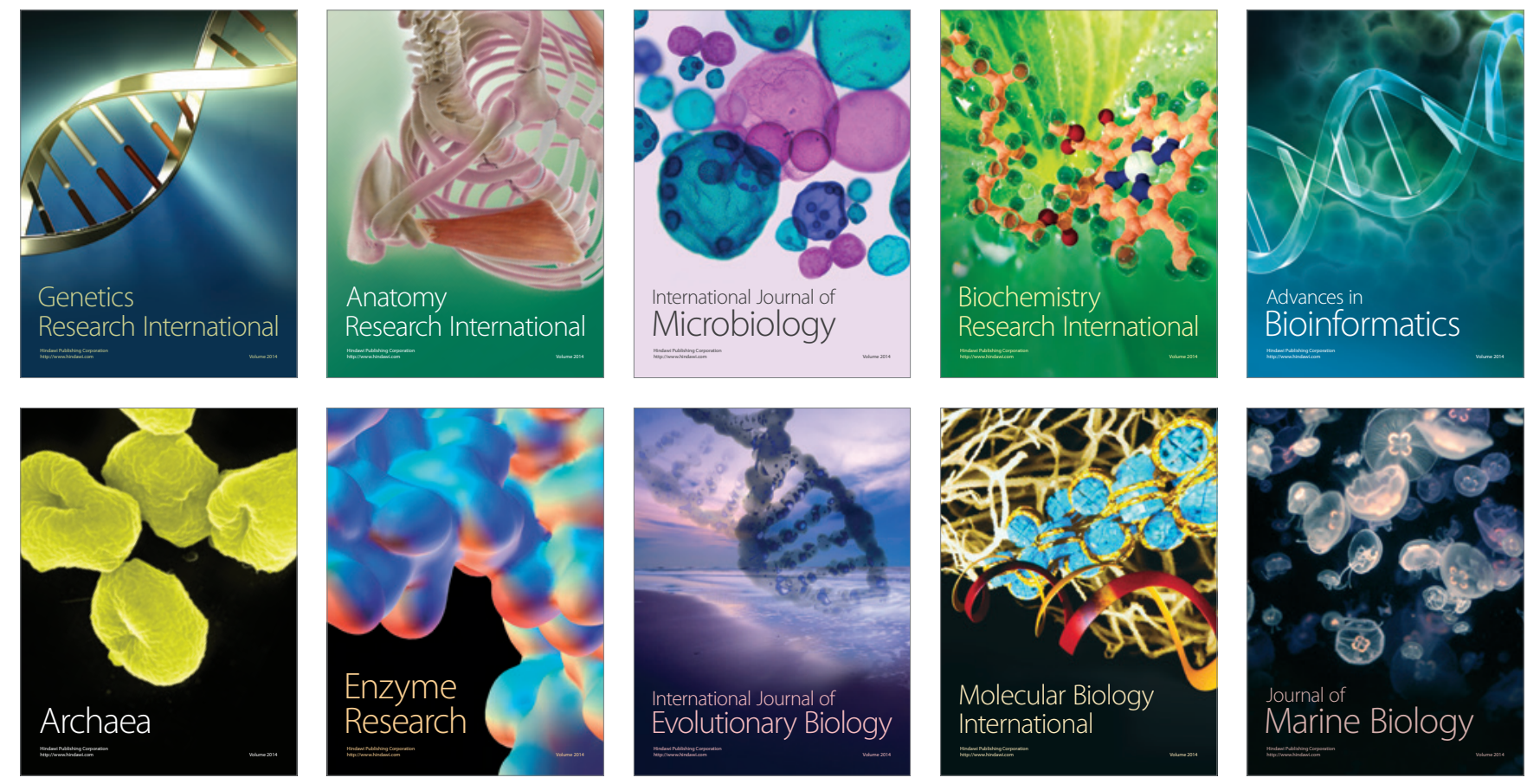